\title{
RESECCIÓN MICROQUIRÚRGICA DE GLIOBLASTOMA GUIADA CON FLUORESCEINAA INTRAOPERATORIA: EVALUACIÓN RETROSPECTIVA
}

\author{
Pamela García-Corrochano1,a, Carlos A. Castañeda ${ }^{1, b}$, Enrique Orrego ${ }^{1, c}$, Pedro Deza $^{1, d}$, Hugo Heinicke ${ }^{1, c}$, \\ Sandro Casavilca ${ }^{1, \mathrm{e}}$, Miluska Castillo ${ }^{1, \mathrm{~h}}$, Karen Cortez $^{1, \mathrm{~g}}$, Carolina Belmar $^{1, \mathrm{i}}$, Ketty Dolores ${ }^{1, \mathrm{f}}$, \\ Claudio Flores ${ }^{2, \mathrm{f}}$, Luis Ojeda ${ }^{1, j}$
}

\section{RESUMEN}

\begin{abstract}
Objetivos. Evaluar la influencia del uso de fluoresceína sódica (FLS-Na) en la cirugía del glioblastoma (GB) sobre el grado de resección tumoral y la supervivencia en pacientes atendidos en el Instituto Nacional de Enfermedades Neoplásicas. Materiales y métodos. Se revisó un total de 238 casos de GB atendidos entre los años 2008 y 2013 y se seleccionó 150 casos de GB sometidos a resección quirúrgica, con información clínico-patológica y seguimiento adecuado. Resultados. La media de edad fue 51 años, el $58,7 \%$ de casos presento Karnofsky de al menos 90 . Se administró FLS-Na en 80 casos $(53,3 \%)$ y se obtuvo una resección subtotal y total en 69 (46\%) y 81 (54\%) de los casos, respectivamente. El grupo que recibió FLS-Na obtuvo mayores tasas de resección total que el grupo operado solo con luz blanca $(77,5$ vs $27,1 \%$, $p<0,001)$. La mediana de sobrevida global (SG) fue mayor en el grupo sometido a resección total que a subtotal (17 vs 7 meses, $p<0,001)$. La mediana de SG en los que recibieron FLS-Na fue mayor que en los que no la recibieron $(15,0$ vs 8 meses, $p=0,003)$. Otros factores que afectaron la SG fueron la edad $(p=0,002)$, el Karnofsky $(p=0,052)$ y la administración de radioterapia $(p=0,016)$ y quimioterapia $(p=0,011)$. Conclusiones. La técnica microquirúrgica con administración de FLSNa se asoció con un aumento en la tasa de resecciones totales y de supervivencia.
\end{abstract}

Palabras clave: Glioblastoma; Fluoresceína; Microcirugia; Análisis de supervivencia (fuente: DeCS BIREME).

\section{MICROSURGICAL RESECTION OF GLIOBLASTOMA GUIDED WITH INTRAOPERATIVE FLUORESCEIN: A RETROSPECTIVE EVALUATION}

\begin{abstract}
Objectives. To evaluate the influence of the use of sodium fluorescein (FLS-Na) in surgery of glioblastoma (GB) on the degree of tumor resection and survival in patients treated at the National Institute of Neoplastic Diseases. Materials and methods. A total of 238 cases of GB treated between 2008 and 2013 were reviewed and 150 cases of GB who underwent surgical resection with clinicopathological information and adequate follow-up were selected. Results. The mean age was 51 years, $58.7 \%$ of the cases presented a Karnofsky score of at least 90. FLS-Na was administered in 80 cases (53.3\%) and a subtotal and total resection was obtained in 69 (46\%) and 81 (54\%) cases, respectively. The group that received FLS-Na obtained higher rates of total resection than the group operated with white light alone $(77.5$ vs $27.1 \%, p<0.001)$. The median overall survival (OS) was higher in the group subject to total compared to subtotal resection (17 vs 7 months, $p<0.001$ ). The median OS in those who received FLS-Na was higher than in those who did not (15.0 vs 8 months, $p=0.003)$. Other factors affecting OS were age $(p=0.002)$, the Karnofsky score $(p=0.052)$ and radiation therapy $(p=0.016)$ and chemotherapy $(p=0.011)$. Conclusions. The microsurgical technique with administration of FLS-Na was associated with an increase in the rate of total resection and survival.
\end{abstract}

Key words: Glioblastoma; Fluorescein; Microsurgery; Survival analysis (source: MeSH NLM).

\section{INTRODUCCIÓN}

El glioblastoma (GB) representa la neoplasia cerebral más frecuente (alrededor de la mitad de los tumores del cerebro) y la de mayor mortalidad.
Es más frecuente entre la quinta y sexta década de la vida y tiene una ligera predisposición por el sexo masculino ${ }^{(1,2)}$. La tasa de incidencia de los tumores del sistema nervioso central (SNC) en Estados Unidos de Norteamérica es alrededor de 11,4 casos

\footnotetext{
Instituto Nacional de Enfermedades Neoplásicas. Lima, Perú.

Oncosalud, AUNA. Lima, Perú.

Médico cirujano; ' Oncólogo clínico, magíster en Genética y Biología Celular, magíster en oncología avanzada; ${ }^{\mathrm{c}}$ neurocirujano, doctor en Medicina ${ }^{\mathrm{d}}$ neurocirujano; ${ }^{\mathrm{e}}$ médico patólogo; ${ }^{\mathrm{f}}$ bioestadístico; ${ }^{\mathrm{g}}$ biólogo; ${ }^{\mathrm{h}}$ toxicóloga ; ${ }^{\mathrm{i}}$ Biotecnóloga, $\mathrm{PhD} ;{ }^{\mathrm{j}}$ neurocirujano, magíster en Medicina.

Recibido: : 20-10-14 Aprobado: 18-03-15
}

Citar como: García-Corrochano P, Castañeda CA, Orrego E, Deza P, Heinicke H, Casavilca S, et al. Resección microquirúrgica de glioblastoma guiada con fluoresceína intraoperatoria: evaluación retrospectiva. Rev Peru Med Exp Salud Publica. 2015;32(3):471-8. 
por 100000 personas/año, y representa el $2,4 \%$ de las muertes por cáncer ${ }^{(3)}$. Mientras que para Lima Metropolitana la tasa de incidencia estandarizada es de 7,0 casos por 100000 personas/año y representa el $3,4 \%$ de las muertes por cáncer ${ }^{(4)}$. La denominación de tumores del SNC incluye tumores del cerebro (alrededor del total), meninges y medula espinal. Estudios epidemiológicos encuentran diferencias en la incidencia de GB según la etnia/raza de la población y sugieren que la incidencia es menor en población latina que en caucásica. Estas diferencias pueden deberse a diferencias en la frecuencia de alelos de susceptibilidad a desarrollar GB descritos para diferentes etnias/razas. Sin embargo, no existe información sobre el comportamiento ni incidencia de esta neoplasia en población peruana ${ }^{(5-8)}$.

El grado de resección de la lesión se asocia con una mayor supervivencia (9). Sin embargo, hasta hace pocos años la resección total del tumor se realizaba con éxito en la minoría de los casos debido a la similitud entre la apariencia del tumor y del parénquima cerebral circundante y debido al comportamiento difuso infiltrante en los gliomas de alto grado. Resecciones extremadamente agresivas pueden producir daño neurológico severo, mientras que resecciones menos agresivas pueden dejar enfermedad residual significativa ${ }^{(10)}$.

La cirugía guiada con altas dosis (AD) de fluoresceína sódica (FLS-Na) es una técnica neuroquirúrgica estándar que incluye la administración intravenosa de este compuesto que se concentra en áreas dañadas de la barrera hematoencefálica (BHE) como la que se encuentra en el área tumoral, su concentración se correlaciona en forma directa con el número de células neoplásicas. Esta técnica permite diferenciar el tejido neoplásico por remover, del tejido cerebral que debe ser preservado. Sin embargo, tiene limitaciones en relación a la subjetiva interpretación de positividad en los márgenes tumorales (región con captación no intensa), la ausencia de captación en gliomas que conservan la BHE y la captación en procesos inflamatorios como infecciones y necrosis ${ }^{(9,11-14)}$. La dosis que administrar se calculó luego de experimentos in vivo con tumores cerebrales en roedores y una serie de cinco pacientes con resección de glioma ${ }^{(15)}$.

El pobre pronóstico asociado al GB lo convierte en un problema epidemiológico a nivel mundial. Los pobres resultados históricos que se observan en pacientes peruanos nos motivaron a incorporar la técnica con AD FLS-Na a la rutina del Instituto Nacional de Enfermedades Neoplásicas (INEN) del Perú desde el año 2010. El propósito de este estudio retrospectivo ha sido evaluar la influencia la técnica en mención sobre el grado de resección tumoral y la sobrevida en pacientes con GB atendidos en el INEN.

\section{MATERIALES Y MÉTODOS}

\section{PACIENTES}

Se revisó los casos que acudieron al INEN entre los años 2008 y 2013 y se identificó 238 casos nuevos con diagnóstico clínico de GB atendidos en él; se seleccionaron 158 pacientes con resultado anatomopatológico de GB (OMS) sometidos a resección quirúrgica (total o subtotal) y con información clínica y seguimiento adecuado. Posteriormente, se excluyeron aquellos tumores localizados en ganglios basales y en fosa posterior; así como a aquellos fallecidos por complicación posoperatoria. De esta forma, se realizó el análisis en una muestra de 150 pacientes.

La información se obtuvo de las historias clínicas almacenadas en el archivo de historias clínicas e incluyó: edad, sexo, estado de rendimiento Karnofsky inicial, localización tumoral, tipo de resección, convulsiones, focalización neuronal, tratamiento adyuvante, recurrencia, evolución posoperatoria, fecha de fallecimiento o de última consulta y fecha de progresión de enfermedad.

\section{EVALUACIÓN RADIOLÓGICA}

Todos los pacientes fueron sometidos a control de imágenes cerebrales posoperatorio dentro de las $72 \mathrm{~h}$ para determinar la extensión/volumen del tumor residual (total o subtotal) y se les realizó el seguimiento de supervivencia (Figura 1).

\section{PROCEDIMIENTO QUIRÚRGICO}

La técnica quirúrgica incluyó la administración de $20 \mathrm{mg} / \mathrm{kg}$ de FLS-Na (ampolla Aka-Fluor 10\% Medifarma S.A.) a través de la línea de una vena periférica antes de la incisión de la duramadre, en forma similar al descrito por Shinoda et al. (12). La resección fue realizada aproximadamente a los 10 min luego de la administración de fluoresceína (Figura 2). El tumor fue removido en bloque en la mayoría de los casos o fue removida pieza a pieza usando un aspirador ultrasónico, en algunos casos. Se continuó con la resección hasta que el tejido fijado con fluoresceína fue removido totalmente y el parénquima cerebral fue visto. Ochenta pacientes $(n=80)$ recibieron FLS-Na y 70 fueron sometidos al procedimiento de microcirugía 

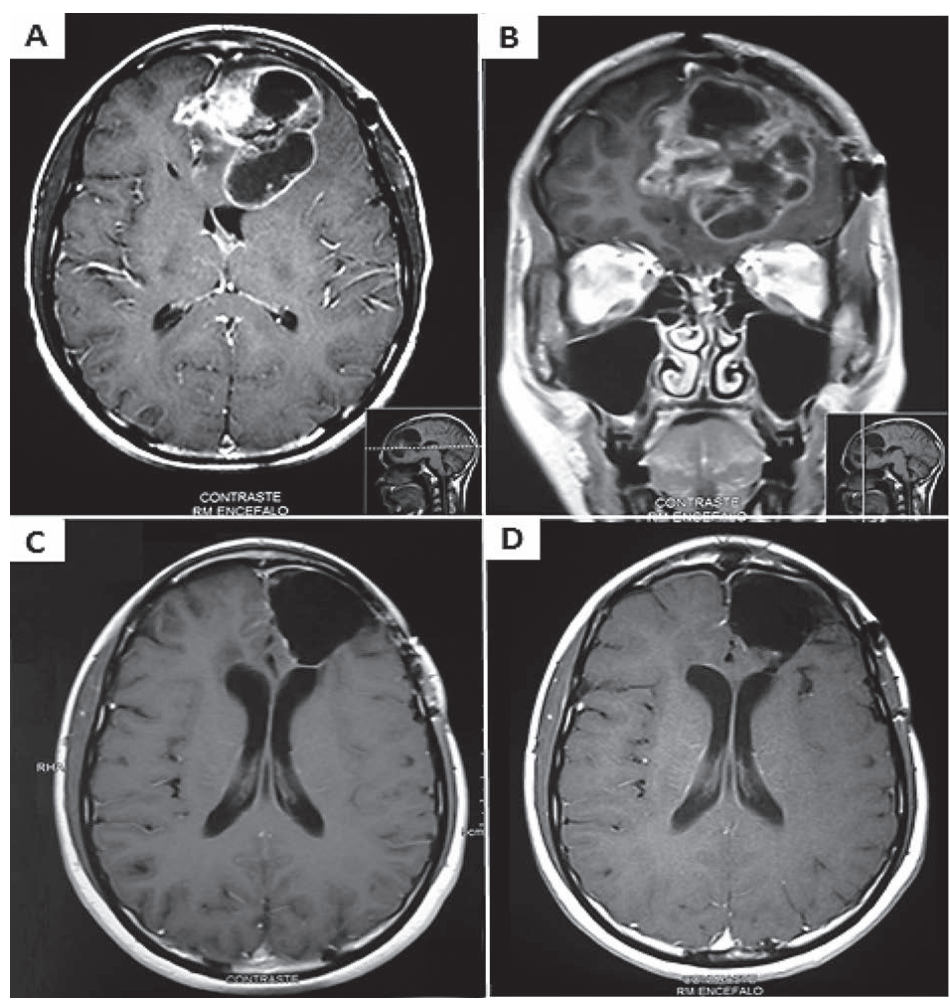

Figura 1. (A) RM Encéfalo T1- con contraste preoperatorio se evidencia tumor sólido de bordes irregulares captador de contraste con componente quístico frontal izquierdo. Desviación de la línea media y compresión del ventrículo lateral izquierdo con efecto de masa: imagen axial. (B) Imagen coronal. (C) RM encéfalo T1-con contraste posoperatorio luego de un mes que demuestra los cambios luego de la cirugía, no evidencia realce de contraste. (D) RM encéfalo T-1 con contraste posoperatorio control a los 3 años de la cirugía, no evidencia realce de contraste

estándar con iluminación de luz blanca. No se utilizó navegación, mapeo intraoperatorio ni luz de microscopio modificada.

\section{PROCEDIMIENTO POSOPERATORIO}

Todos los pacientes fueron enviados a tratamiento con RT y quimioterapia posoperatoria dentro de los 30 días posteriores a la cirugía. La radiación a la que fueron sometidos los pacientes fue determinada por el fraccionamiento convencional hasta una dosis total de $6000-6500$ cGy.

\section{ANÁLISIS ESTADÍSTICO}

Para la realización de la comparación de proporciones de muestras independientes se empleó la prueba Z. El tiempo de seguimiento para estimar la sobrevida global comprende desde la fecha de cirugía hasta la fecha de muerte o último control. El tiempo de seguimiento para

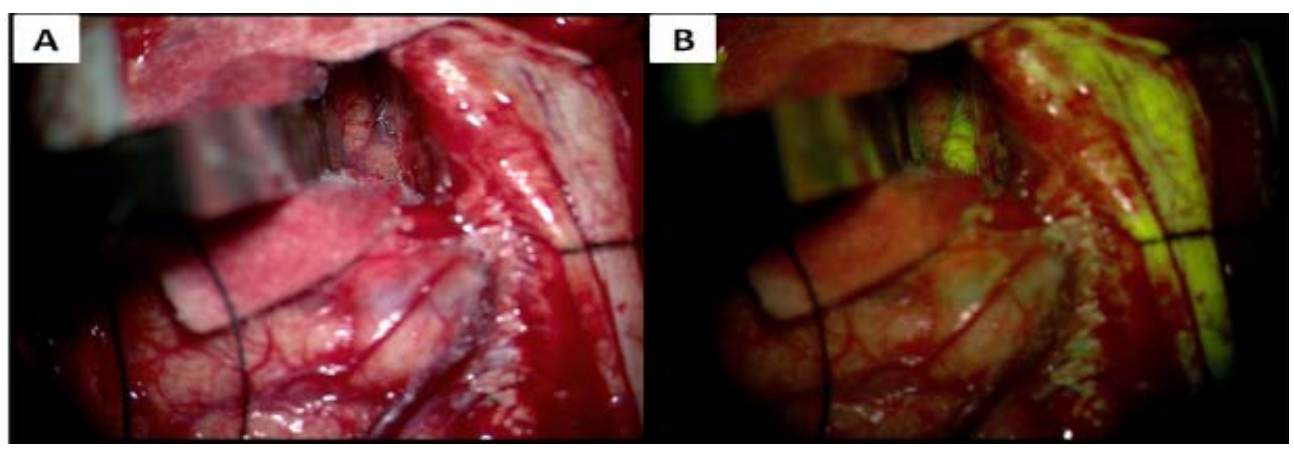

Figura 2. (A) Parénquima cerebral sin efecto de la adición de FLS-Na. (B) Parénquima cerebral con efecto de FLS-Na y la tecnología de realce con luz azul que brinda el microscopio OPMI PENTERO 900. 
estimar la sobrevida libre de progresión desde la fecha de cirugía hasta la fecha de recurrencia o último control. Las curvas de sobrevida se calcularon por el método de Kaplan-Meier, y la comparación entre curvas de diferentes categorías de las características se probó mediante log-rank test. Para el análisis multivariado de supervivencia se empleó la regresión de Cox. Se evaluó el supuesto de riesgos proporcionales. Se consideró una diferencia significativa al valor de $p<0,05$. Se usó el programa estadístico STATA versión 13.

\section{CONSIDERACIONES ÉTICAS}

La base de datos creada no incluyó la identidad de los pacientes y la información obtenida se mantuvo en completa confidencialidad. Este proyecto fue aprobado por el Comité Revisor de Protocolo y el Comité de Ética del Instituto Nacional de Enfermedades Neoplásicas.

\section{RESULTADOS}

La población de estudio la conformaron 150 pacientes con GB. La media de edad fue 51 años (879 años), incluyó 89 hombres (59,3\%), y la escala de rendimiento Karnofsky inicial fue de al menos 90 en el $96 \%$ de los casos. El 34,7\% presentó convulsiones y el $53,3 \%$ síntomas de focalización. La ubicación tumoral más frecuente fue: frontal (35\%), temporal $(29,4 \%)$, parietal $(21,7 \%)$ u occipital (10\%). La mediana de diámetro mayor de las lesiones fue de $5 \mathrm{~cm}(1-8,4 \mathrm{~cm})$.

Se administró AD FLS-Na en 80 casos (53,3\%). Se obtuvo una resección subtotal en 69 (46\%) y total en $81(54 \%)$ casos. Se administró tratamiento adyuvante con radioterapia en 95 casos (63,3\%). La quimioterapia adyuvante fue utilizada en 79 casos

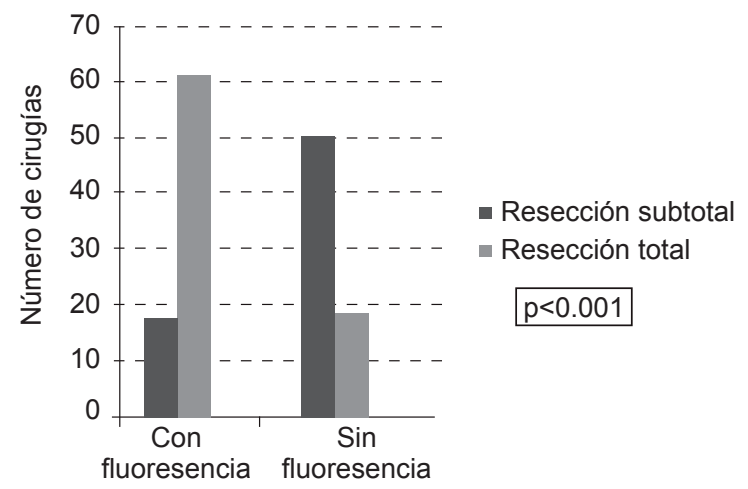

Figura 3. Influencia del uso de FLS-Na sobre el grado de resección tumoral.
Tabla 1. Características clínico-patológicas

\begin{tabular}{|c|c|c|}
\hline Variable & $\mathbf{n}$ & (\%) \\
\hline \multicolumn{3}{|l|}{ Edad (años) } \\
\hline Mediana (rango) & $51(8-79)$ & \\
\hline \multicolumn{3}{|l|}{ Sexo } \\
\hline Hombre & 89 & $(59,3)$ \\
\hline Mujer & 61 & $(40,7)$ \\
\hline \multicolumn{3}{|l|}{ Escala de Karnofsky } \\
\hline 70 & 22 & $(14,7)$ \\
\hline 80 & 40 & $(26,7)$ \\
\hline 90 & 82 & $(54,7)$ \\
\hline 100 & 6 & $(4,0)$ \\
\hline \multicolumn{3}{|l|}{ Diagnóstico histológico } \\
\hline Glioblastoma & 104 & $(69,3)$ \\
\hline Glioblastoma cel. Pequeñas & 8 & $(5,3)$ \\
\hline Glioblastoma cel. Gigantes & 11 & $(7,3)$ \\
\hline $\begin{array}{l}\text { Glioblastoma Comp. } \\
\text { oligodendroglial }\end{array}$ & 18 & $(12,0$ \\
\hline Glioblastoma PNET & 5 & $(3,3)$ \\
\hline Gliosarcoma & 4 & $(2,7)$ \\
\hline \multicolumn{3}{|l|}{ Localización } \\
\hline Frontal & 63 & $(35,0)$ \\
\hline Temporal & 53 & $(29,4)$ \\
\hline Occipital & 18 & $(10,0)$ \\
\hline Parietal & 39 & $(21,7)$ \\
\hline $\begin{array}{l}\text { Pineal/cuerpo calloso/ } \\
\text { intraventricular }\end{array}$ & 7 & $(3,9)$ \\
\hline \multicolumn{3}{|l|}{ Tamaño (cm) } \\
\hline Mediana (rango) & $5(1-8,4)$ & \\
\hline \multicolumn{3}{|l|}{ Sintomatología } \\
\hline Convulsiones & 52 & $(34,7)$ \\
\hline Focalización neurológica & 80 & $(53,3)$ \\
\hline \multicolumn{3}{|l|}{ Fluoresceína } \\
\hline No & 70 & $(46,7)$ \\
\hline Sí & 80 & $(53,3)$ \\
\hline \multicolumn{3}{|l|}{ Tipo de cirugía } \\
\hline Cirugía total & 81 & $(54,0)$ \\
\hline Cirugía subtotal & 69 & $(46,0)$ \\
\hline \multicolumn{3}{|l|}{ Adyuvancia } \\
\hline Radioterapia & 95 & $(63,3)$ \\
\hline Quimioterapia & 79 & $(52,7)$ \\
\hline Temozolomida & 75 & $(92,6)$ \\
\hline Carmustina & 3 & $(3,7)$ \\
\hline Bevacizumab & 3 & $(3,7)$ \\
\hline
\end{tabular}

$(52,7 \%)$ y constó de temozolomida monodroga en 75 casos (Tabla 1).

Los casos con nivel de rendimiento Karnofsky, de al menos 90, tuvieron mayores tasas de resección total $(60,2$ versus $45,2 \%, p=0,034)$. El grupo que recibió FLS$\mathrm{Na}$ obtuvo mayor resección total que el grupo operado solo con luz blanca (77,5 versus $27,1 \%, p<0,001)$ (Figura 3). 


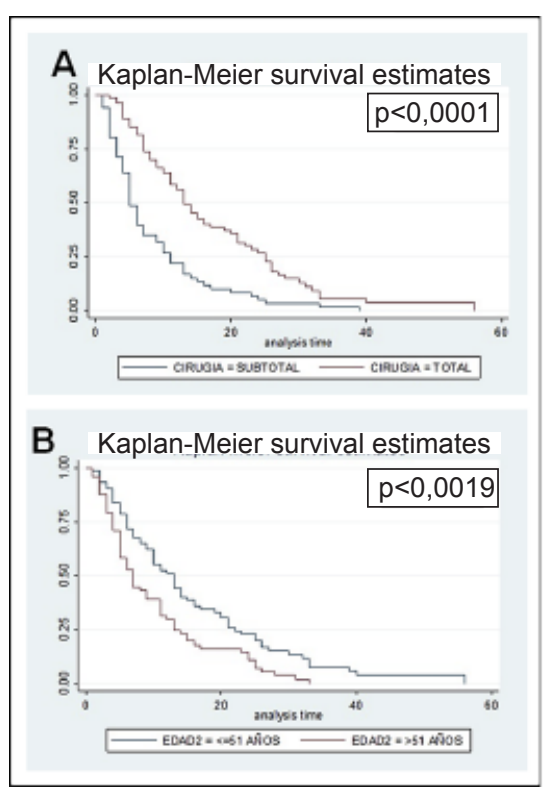

Figura 4. Curvas de Kaplan-Meier de sobrevida libre de progresión según tipo de resección (total vs. sub-total) (A) y edad (B).

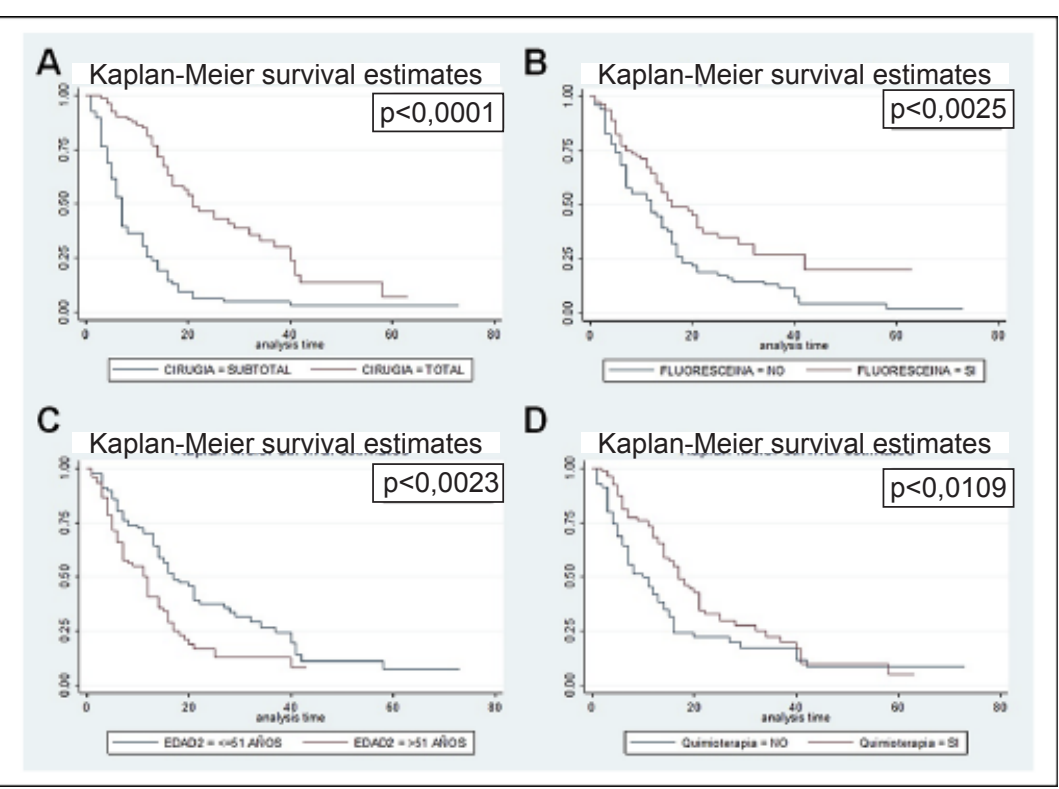

Figura 5. Curvas de Kaplan-Meier de supervivencia global según tipo de resección (total vs. subtotal) (A), uso de fluoresceína (B), edad (C) y quimioterapia (D).
La mediana de seguimiento fue de 13 meses, el $92,7 \%$ de casos progresó durante el seguimiento y el $77,3 \%$ de los pacientes falleció.

La mediana de sobrevida libre de progresión (SLP) en la población total fue de 9 meses, en la que recibió FLS$\mathrm{Na}$ fue de 10 meses y en las que no recibió FLS-Na fue de 7 meses $(p=0,059)$. La mediana de SLP fue de 11,5 meses en menores o iguales a 50 años y 7 meses en mayores de 51 años $(p=0,002)$. La mediana de SLP tanto para varones y mujeres fue 9 meses $(p=0,965)$. La mediana de SLP fue de 8,5 meses en pacientes con nivel de rendimiento Karnofsky $70-80$ y de 9 meses en pacientes con nivel de rendimiento Karnofsky 90$100(p=0,167)$. La mediana de SLP para la población sometida a resección total y a subtotal fueron de 15 y 7 meses $(p=0,001)$, respectivamente. La mediana de SLP en pacientes que recibieron o no recibieron radioterapia (RT) fue de 10 y 7 meses $(p=0,455)$, respectivamente. La mediana de SLP en pacientes que recibieron o no recibieron quimioterapia fue de 11 y 7 meses $(p=0,350)$, respectivamente (Figura 4 ). El análisis multivariado encuentra que solo la edad $(p=0,013)$ y el tipo de resección $(p<0,001)$ se asoció a SLP.

La mediana de sobrevida global (SG) en la población total fue de 13 meses. La mediana de SG fue de 15,5 meses en menores o iguales a 51 años y 10 meses en mayores de 51 años $(p=0,002)$. La mediana de SG fue de 12 para varones y 14 para mujeres $(p=0,059)$. La mediana de SG fue de 12 meses en pacientes con Karnofsky $70-80$ y de 13 meses en pacientes con nivel de rendimiento Karnofsky 90$100(p=0,051)$. La mediana de SG para la población sometida a resección total y a subtotal fueron de 17 y 7 meses $(p<0,001)$, respectivamente. La mediana de $S G$ en pacientes que recibieron o no recibieron RT fue de 15 y 8 meses $(p=0,016)$, respectivamente. La mediana de SG en pacientes que recibieron o no recibieron quimioterapia fue de 16 y 8 meses $(p=0,011)$, respectivamente. La mediana de SG en la población que recibió FLS-Na fue de 15 meses y en las que no recibió FLS-Na fue de 8 meses $(p=0,002)$ (Figura 5). El análisis multivariado encuentra que solo edad $(p=0,007)$ y el uso de fluoresceína $(p=0,002)$ se asoció a SG.

\section{DISCUSIÓN}

A nuestro conocimiento, este artículo es el primer esfuerzo multidisciplinario de esta década en evaluar el impacto de la incorporación de las nuevas técnicas de tratamiento en la sobrevida de pacientes, así mismo, se describe el comportamiento clínico del GB en una población peruana.

La revisión exhaustiva de información del INEN, que atiende aproximadamente el $40 \%$ de los pacientes con cáncer, indica que han acudido 238 casos nuevos de GB a esta institución durante los 6 años que se evalúan. El registro de cáncer de Lima- Metropolitana indica una tasa de incidencia total de tumores del SNC $(7,0$ casos por 100000 personas/año) menor que la encontrada 
en países de raza caucásica. Esta información podría indicar que la raza/etnia influye en forma significativa en el comportamiento del GB. Esta información tiene implicancia en la toma de decisiones en el instituto y en las políticas de salud a nivel nacional ${ }^{(4,8)}$.

El análisis de esta información encuentra que la edad se asocia a SLP; y la edad, sexo, nivel de rendimiento Karnofsky, uso de RT y uso de quimioterapia se asociaron a SG. Estas asociaciones han sido descritas por diversos autores e indica la relevancia del estado del huésped en el éxito de los tratamientos. El mejor pronóstico encontrado para los varones está probablemente asociado con su relación con las otras variables, pues la asociación desaparece en el análisis multivariado ${ }^{(9,12,16,17)}$.

Un nivel de rendimiento karnofsky de al menos 90 se asoció también con un mayor porcentaje de resecciones totales. Se encontró también la asociación entre el grado de resección y la sobrevida de los pacientes, confirmando que el objetivo de la cirugía debe ser el obtener el mayor grado de resección posible. Diversos estudios retrospectivos con tamaño de muestra escaso encuentran que la extensión de la resección quirúrgica para el GB tiene un efecto significativo sobre el pronóstico ${ }^{(18,19)}$. Lacroix et al. evaluaron una serie retrospectiva de 416 GB y encontraron un incremento de 3,2 meses en la sobrevida asociado a resección $\geq 98 \%$ del tumor (16); Stummer et al. reportaron un incremento de 4,9 meses en la sobrevida para aquellos pacientes sin enfermedad residual una serie de 243 casos de $\mathrm{GB}{ }^{(9)}$.

La incorporación de FLS-Na se asocia con un mayor porcentaje de resecciones totales $(77,5$ vs $27,1 \%)$. Así mismo, el análisis de sobrevida encontró que la incorporación de FLS-Na se asocia con una mayor sobrevida. La cirugía guiada por fluorescencia es un procedimiento que puede ser fácilmente realizado y no necesita tiempo ni instrumentación adicional. Diversos estudios encuentran que la utilización de FLS-Na se asocia con un incremento en el grado de extensión de la resección. Koc et al. evaluaron una serie retrospectiva de 80 casos de GB en la que 47 utilizaron FLS-Na y encontraron que el uso de fluoresceína se asoció con un incremento del $28 \%$ en las tasas de resección total mayor, sin embargo, no encontró asociación con un incremento en la sobrevida global (17). Chen et al. evalúa 22 pacientes con GB sometidos a resección tumoral y encuentra que los 10 casos que recibieron FLS-Na presentaron mayores tasas de resección total $(p=0,001)$ y obtuvieron mayores sobrevidas libres de progresión $(p=0,033)$. Shinoda et al. realizaron la resección microquirúrgica estándar después de la administración intravenosa de fluoresceína de sodio. En este estudio, la tasa de resección total incrementó en el grupo con administración de fluoresceína $(84,4 \%, 27 / 32)$, versus sin este procedimiento $(30,1 \%, 22 / 73)^{(12)}$.

Algunas series retrospectivas que evalúan otro marcador de fluorescencia como ácido 5aminolevulinico (5- ASA) también han encontrado su asociación con incremento en tasas de resección y sobrevida ${ }^{(18,19)}$. El único estudio randomizado Fase III que evalúa la administración de 5- ASA versus luz blanca en 322 casos con sospecha de glioma maligno, encuentra que el marcador se asocia con un incremento en la tasa de resección amplia (65 vs 36\%), así como a un incremento en la sobrevida libre de progresión $(5,1$ vs 3,6 meses); sin embargo, no encontró diferencias para la sobrevida global (15,2 versus 13,5 meses) y algunos críticos sugieren que las diferencias encontradas pueden deberse a la corta sobrevida del grupo control ${ }^{(9)}$. Finalmente, un metaanálisis con diez estudios que evalúan la utilidad del uso de 5- ASA en la resección tumoral, encuentra que su uso se asocia con un incremento en las tasas de resección completa y en la sobrevida ${ }^{(20)}$. La 5- ASA se administra vía oral y tiene el inconveniente de requerir un mayor periodo entre la administración y la visualización; mientras que la administración intravenosa de las altas dosis requeridas de AD FLS-Na no está exento de toxicidad para el paciente ${ }^{(21)}$.

Como una limitación del estudio se puede mencionar que se trata de una evaluación retrospectiva observacional y así las asociaciones encontradas pueden deberse a la influencia de parámetros externos como el uso del aspirador ultrasónico en algunos casos o a la ausencia de decisión aleatoria de utilizar AD FLS-Na por parte del neurocirujano ${ }^{(22)}$. Una fortaleza del estudio es que debido al rol docente de los cirujanos del instituto, todos los miembros del Departamento de Neurocirugía realizan la técnica quirúrgica en forma similar. Así mismo, la información que se presenta es la obtenida en pacientes peruanos evaluados en forma rutinaria y, de esta forma, los resultados se pueden aplicar a nuestros pacientes.

El pobre pronóstico asociado al GB nos exige implementar nueva metodología para el incremento de las tasas de RT en nuestros pacientes. Así, hemos recientemente incorporado el microscopio OPMI Pentero 900 -Zeiss a la sala de neurocirugía. Este equipo permite reducir la cantidad de FLS-Na inyectada y realizar la cirugía guiada por imágenes con la inyección de datos multivisión, así como la visualización digital integrada con cámara de alta definición ${ }^{(23)}$. 
Se concluye que, en forma similar a otros estudios previamente reportados, el uso de FLS-Na estuvo asociado con tasas mayores de resección completa y a un incremento en la sobrevida que la microcirugía estándar. Otros factores como edad, sexo, estado de performance, uso de tratamiento de radiación y quimioterapia influyen en la sobrevida de los pacientes.

Contribuciones de autoría: CAC, PGC participaron en la concepción y diseño del artículo; CAC, PGC, KC, MC participaron en la recolección y obtención de resultados; CAC, PGC, participó en el análisis e interpretación de datos; MCG, PGC, SC y MC participaron en la redacción del artículo; y CAC, $\mathrm{EO}, \mathrm{PD}, \mathrm{HH}, \mathrm{LO}$ participaron en la revisión crítica del artículo; CAC, EO, PGC realizaron la aprobación de su versión final; PGC obtuvo el financiamiento; CF participó en la asesoría estadística.

Fuentes de financiamiento: autofinanciado.

Conflictos de interés: los autores declaran no tener conflictos de interés.

\section{REFERENCIAS BIBLIOGRÁFICAS}

1. Jemal A, Siegel R, Ward E, Hao Y, Xu J, Murray T, et al. Cancer statistics, 2008. CA Cancer J Clin. 2008;58(2):71-96. doi: 10.3322/CA.2007.0010.

2. Gordon-Firing S, Garbino Bonomi C. Clasificación e histogénesis de los tumores primitivos del SNC. En: Tratamiento de los tumores primitivos del sistema nervioso central: nuestra experiencia desde 1975 a 1993. Montevideo: Oficina del Libro AEM; 1995. p. 19-29.

3. Louis DN, Ohgaki H, Wiestler OD, Cavenee WK, Burger PC, Jouvet A, et al. The 2007 WHO classification of tumours of the central nervous system. Acta Neuropathol. 2007;114(2):97109. Epub 2007 Jul 6.

4. Instituto Nacional de Enfermedades Neoplasicas. Registro de Cáncer de Lima Metropolitana. Volumen IV Estudio de incidencia y mortalidad (2004-2005).Lima: INEN; 2014.

5. Dubrow R, Darefsky AS. Demographic variation in incidence of adult glioma by subtype, United States, 1992-2007. BMC Cancer. 2011;11:325. doi: 10.1186/1471-2407-11-325.

6. Preston Martin S. Epidemiology. En: Berger M, Wilson CB, eds. The gliomas. Philadelphia: WB Saunders; 1999. p. 2-11.

7. Kleihues P, Cavenee K; World Health Organization Classification of Tumours. Pathology and genetics. Tumours of the nervous system. Wasington DC: WHO/IARC Press; 2000.
8. Fisher JL, Schwartzbaum JA, Wrensch $\mathrm{M}$, Wiemels JL. Epidemiology of brain tumors. Neurol Clin. 2007;25(4):86790, vii.

9. Stummer W, Reulen HJ, Meinel T, Pichlmeier U, Schumacher W, Tonn $\mathrm{JC}$, et al. Extent of resection and survival in glioblastoma multiforme: identification of and adjustment for bias. Neurosurgery. 2008;62(3):56476; discussion 564-76. doi: 10.1227/01. neu.0000317304.31579.17.

10. Ohgaki H, Kleihues P. Genetic pathways to primary and secondary glioblastoma. Am J Pathol. 2007;170(5):1445-53.

11. Stummer W, Novotny A, Stepp H, Goetz C, Bise K, Reulen HJ. Fluorescence-guided resection of glioblastoma multiforme by using 5-aminolevulinic acid-induced porphyrins: a prospective study in 52 consecutive patients. J Neurosurg. 2000 Dec;93(6):1003-13.

12. Shinoda J, Yano $H$, Yoshimura $S$, Okumura A, Kaku Y, Iwama T, et al. Fluorescence-guided resection of glioblastoma multiforme by using high-dose fluorescein sodium. Technical note. J Neurosurg. 2003 Sep;99(3):597-603.

13. Stummer W, Pichlmeier U, Meinel T, Wiestler OD, Zanella F, Reulen HJ. Fluorescence-guided surgery with 5-aminolevulinic acid for resection of malignant glioma: a randomised controlled multicentre phase III trial. Lancet Oncol. 2006;7(5):392-401.
14. Willems PW, Taphoorn MJ, Burger $\mathrm{H}$, Berkelbach van der Sprenkel JW, Tulleken CA. Effectiveness of neuronavigation in resecting solitary intracerebral contrast-enhancing tumors: a randomized controlled trial. J Neurosurg. 2006;104(3):360-8.

15. Kabuto M, Kubota T, Kobayashi H, Nakagawa T, Ishii $\mathrm{H}$, Takeuchi $\mathrm{H}$, et al. Experimental and clinical study of detection of glioma at surgery using fluorescent imaging by a surgical microscope after fluorescein administration. Neurol Res. 1997;19(1):9-16.

16. Lacroix M, Abi-Said D, Fourney DR, Gokaslan ZL, Shi W, DeMonte F et al. A multivariate analysis of 416 patients with glioblastoma multiforme: prognosis, extent of resection, and survival. J Neurosurg. 2001 Aug;95(2):190-8

17. Koc K, Anik I, Cabuk B, Ceylan S. Fluorescein sodium-guided surgery in glioblastoma multiforme: a prospective evaluation. Br J Neurosurg. 2008 Feb;22(1):99-103. doi: $10.1080 / 02688690701765524$.

18. Della Puppa A, Ciccarino P, Lombardi G, Rolma G, Cecchin D, Rossetto M. 5-Aminolevulinic acid fluorescence in high grade glioma surgery: surgical outcome, intraoperative findings, and fluorescence patterns. Biomed Res Int. 2014;2014:232561. doi: $10.1155 / 2014 / 232561$.

19. Diez Valle R, Slof J, Galván J, Arza C, Romariz C, Vidal C. [Observational, retrospective study of the effectiveness 
of 5-aminolevulinic acid in malignant glioma surgery in Spain (The VISIONA study)]. Neurologia. 2014;29(3):1318. doi: 10.1016/j.nrl.2013.05.004. [Artículo en español]

20. Zhao S, Wu J, Wang C, Liu $\mathrm{H}$, Dong X, Shi C, et al. Intraoperative fluorescence-guided resection of high-grade malignant gliomas using 5-aminolevulinic acid-induced porphyrins: a systematic review and meta-analysis of prospective studies. PLoS One. 2013 May 28;8(5):e63682. doi: 10.1371/journal.pone.0063682.
21. Li Y, Rey-Dios R, Roberts DW, Valdes PA, Cohen-Gadol AA. Intraoperative fluorescence-guided resection of highgrade gliomas: a comparison of the present techniques and evolution of future strategies. World Neurosurg. 2014 Jul-Aug;82(1-2):175-85. doi: 10.1016/j.wneu.2013.06.014.

22. Hammoude A. Edge detection in ultrasound images based on differential tissue attenuation rates. Ultrasonic imaging. 1999;21(1):31-42.

23. Kuroiwa T, Kajimoto Y, Ohta T. Development of a fluorescein operative microscope for use during malignant glioma surgery: a technical note and preliminary report. Surg Neurol. 1998 Jul;50(1):41-8.

Correspondencia: Carlos A. Castañeda Altamirano

Dirección: Av. Angamos Este 2520 -

Surquillo, Lima, Perú

Teléfono: (+511) 992157220

Correo electrónico:

carloscastanedaaltamirano@yahoo.com

\section{Visite nuestra página en Facebook, www.facebook.com/rpmesp infórmese sobre los eventos y los nuevos contenidos de la Revista Peruana de Medicina Experimental y Salud Pública}

Notificaciones

Estadisticas

Herramientas de publicación

Configuración Ayuda .

\section{REVISTA PERUANADE MEDICINA EXPERIMENTAL Y SALUD PÚBLICA \\ Difundiendo investigación en salud}

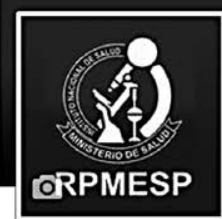

Revista Peruana de Medicina Experimental y Salud Pública (OFICIAL) Comunidad (?])

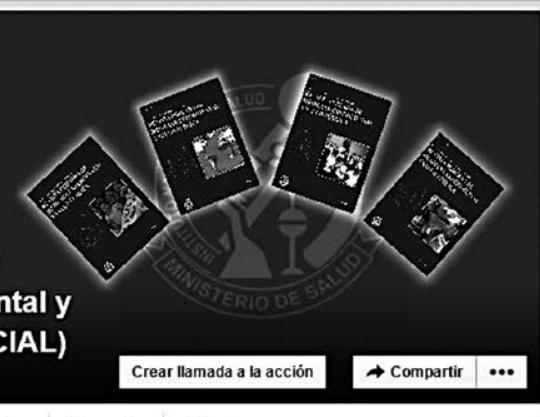

Biografia

\begin{tabular}{l|l|l} 
Información & Fotos Me gusta Más .
\end{tabular}

s.ts 2843 Me gusta +32 esta semana

tall 212 personas alcanzadas esta semana

4. Invitar a amigos a que indiquen que les gusta la..

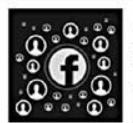

Fortalece ahora tu marca Acércate a los fans de tu negocio e impulsa tus ventas.

\section{Promocionar pégina}

INFORMACIÓN

La Revista Peruana de Medicina Experimentaly Salud Publica es el órgano oficial de difusión cientifica del Instituto Nacional de Salud

nttp:/Wwww ins.gob pe/f.

$$
\text { Promocionar sitio meb }
$$

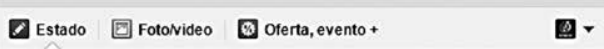

(3) ¿Qué estuviste haciendo?

(30) Revista Peruana de Medicina Experimental y Salud Pública (OFICIAL)

Publicado por rpmesp.informes@gmail.com [?] · Ayer a las 8:43 - ه

Estimados amigos:

Debido al incremento de la investigación de ciencias básicas en nuestro

medio el comité editor ha considerado hacer un numero dedicado

"CIENCIAS BASICAS Y SU CONTRIBUCION A LA PRACTICA CLINICA:

LA EXPERIENCIA PERUANA 2015", que se publicará en el cuarto número de la RPMESP.

La fecha limite para la presentación de artículos es el 31 de Julio del

2015. Las instrucciones para la presentación de articulos es:

http://Www.rpmesp.ins.gob.pe/portalweb/InstruccArt.htm

194 personas alcanzadas Promoción no disponible

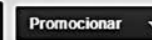

ESTA SEMANA 32 Me gusta de
página 212 Alcance de la
publicación | Reciente 2014 2013 2012 Fundación Ve tu anuncio aqui

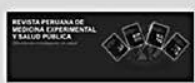

Revista Peruana de Medici... La Revista Peruana de Medic ina Experimental y Salud Public a es el órgano oficial de difusi. B Me gusta $\cdot A 2843$ página Promocionar página mo 